\title{
Osteossíntese mandibular em jiboia (Boa constrictor)
}

\section{Mandibular osteosynthesis in a Boa constrictor snake}

\author{
Jorge Luiz Costa Castro ${ }^{1 *}$; Sérgio Santalucia ${ }^{2}$; José Ricardo Pachaly ${ }^{3}$; \\ José Ademar Villanova Junior'; Verônica Souza Paiva Castro ${ }^{5}$; \\ Daniel Almeida Balthazar ${ }^{6}$; Alex Lucas Spadetti ${ }^{6}$; Wilker Nazareth Souza ${ }^{7}$; \\ Luiz Paulo Luzes Fedullo; ; Alceu Gaspar Raiser ${ }^{8}$
}

\section{Resumo}

Tem-se observado um aumento do achado de determinados animais selvagens em áreas urbanas, em função das alterações ambientais provocadas pelo desmatamento e uso econômico de áreas naturais. Isso causa o desaparecimento das presas usuais e força esses animais, inclusive serpentes, a migrar para áreas urbanizadas, tornando-os vulneráveis aos traumatismos provocados por agressões, atropelamentos e acidentes de captura. As fraturas de mandíbula e maxila são comuns em diversas espécies animais, representando cerca de 3 a $6 \%$ de todas as fraturas ósseas em cães e gatos. $\mathrm{O}$ trauma mandibular ocorre geralmente em consequência de lutas, acidentes veiculares e manejo e/ou contenção inadequados. A mandíbula é um osso chato com diferenças em relação aos ossos longos que devem ser levadas em consideração para o sucesso do tratamento, e a necessidade de manutenção da oclusão e a cobertura muscular mínima são fatores que influenciam a definição do melhor método de redução. Entre os métodos de estabilização se destacam a utilização de fio metálico, pino intramedular, fixador esquelético externo, placa de compressão dinâmica e resina acrílica. A utilização de placas ósseas convencionais é eficiente, mas está relacionada a diversas complicações, tais como a necessidade de elevação da musculatura para sua aplicação e alto risco de lesão às estruturas mandibulares. Este artigo descreve os resultados satisfatórios da aplicação de placa de compressão dinâmica e parafusos na redução de fratura mandibular em uma serpente jiboia (Boa constrictor) do sexo feminino, com peso de $8,0 \mathrm{~kg}$ e comprimento de $1,80 \mathrm{~m}$, atendida na Fundação RIOZOO (Rio de Janeiro, RJ, Brasil).

Palavras-chave: Fratura, ortopedia, animais selvagens, serpente, Boidae

\footnotetext{
${ }^{1}$ Prof. de Técnica Cirúrgica Veterinária da Pontifícia Universidade Católica do Paraná, PUCPR, Programa de Pós-graduação em Medicina Veterinária, PPGMV, Centro de Ciências Rurais, CCR, Universidade Federal de Santa Maria, UFSM, Santa Maria, RS. E-mail: castrojlc@gmail.com

${ }^{2}$ Discente de Mestrado do Programa de Pós-graduação em Medicina Veterinária, PPGMV, CCR/UFSM, Santa Maria, RS. E-mail: santalucia.sergio@gmail.com

${ }_{3}^{3}$ Prof. Titular da Universidade Paranaense, UNIPAR, Umuarama, PR. E-mail: pachaly@uol.com.br

${ }^{4}$ Prof. Assistente III da Pontifícia Universidade Católica do Paraná, PUCPR. E-mail: jose.villanova@pucpr.br

${ }^{5}$ Discente de Doutorado do Programa de Pós-graduação em Medicina Veterinária, PPGMV, CCR/UFSM, Santa Maria, RS. E-mail: castrove@gmail.com

${ }^{6}$ Médicos Veterinários, Fundação Parque Jardim Zoológico da Cidade do Rio de Janeiro, RIOZOO, Rio de Janeiro, RJ. E-mail: danielbalthazar@yahoo.com.br; spadetti@gmail.com; fedullolp@gmail.com

${ }^{7}$ Discente de graduação do curso de Medicina Veterinária, Universidade Castelo Branco, UCB, Rio de Janeiro, RJ. E-mail: wilkervet@gmail.com

${ }^{8}$ Prof. Dr. do Programa de Pós-graduação em Medicina Veterinária, PPGMV, CCR/UFSM, Santa Maria, RS. E-mail: alceu. raiser@gmail.com

* Autor para correspondência
} 


\begin{abstract}
Nowadays are observed an increase in the finding of certain wild animals in urban areas, due to environmental changes caused by deforestation and economic use of natural areas. It causes disappearance of usual prey and forces these animals, including snakes, to migrate to urban areas, becoming vulnerable to injuries caused by aggressions, car accidents and capture. Mandibular and maxillar fractures are common in many animal species, representing about 3-6\% of all bone fractures in dogs and cats. Mandibular trauma usually occurs as a result of fights, car accidents and improper handling and/or restraint, and fractures can be closed or open, clean or contaminated. The jaw is a flat bone with differences from the long bones that should be taken into consideration for successful treatment, being minimal muscle coverage and need to maintain occlusion factors that influence the definition of the best ostheosynthesis method. The methods of stabilization include using intramedullary pins, wires, external skeletal fixation, bone plate, and acrylic resin. Conventional bone plates are efficient but related to some complications, such as the necessity of muscular elevation and high risk of injuries to mandibular structures. This article describes the successful results of the application of plate and screws in the ostheosynthesis of a mandibular fracture in a female Boa constrictor snake with weight of $8.0 \mathrm{~kg}$ and length of $1.80 \mathrm{~m}$, at the RIOZOO Foundation (Rio de Janeiro, RJ, Brazil).
\end{abstract}

Key words: Fracture, orthopedics, wild animals, reptile, Boidae

\section{Introdução}

As fraturas de mandíbula e maxila são comuns em cães e gatos, representando cerca de 3 a $6 \%$ de todas as fraturas ósseas nessas espécies. Os traumas mandibulares ocorrem, geralmente, em consequência de lutas e acidentes veiculares, sendo comumente lesões abertas e contaminadas (LEGENDRE, 2005; PIERMATTEI; FLO; DECAMP, 2009). A mandíbula apresenta algumas peculiaridades em relação aos ossos longos, que devem ser levadas em consideração para o tratamento adequado das fraturas. Nos Squamata, cada hemimandíbula é composta geralmente por sete ossos: dentário, esplenial, esquamosal, coronóide, pré-articular, supra-angular e articular. A região anterior é formada pelo osso dentário, apresentando em sua região dorsal uma crista (crista dentalis) que serve de apoio para os dentes. As duas hemimandíbulas estão conectadas pela sínfese mandibular, estrutura de composição variada que limita o movimento das mesmas mantendo-as unidas funcionalmente (HOCKNULL, 2002).

Anecessidade de manutenção da oclusão e a baixa cobertura muscular são fatores a considerar antes da escolha do método cirúrgico. $\mathrm{O}$ fato de não sustentar peso, entretanto, permite que, em alguns casos não seja necessário usar fixação rígida (LEGENDRE, 2005). As fraturas mandibulares podem também levar a alterações anatomofisiológicas em funções básicas como mastigação e deglutição (RAIMUNDO et al., 2008).

A jiboia (Boa constrictor) é uma grande serpente neotropical que se alimenta de pequenos mamíferos (especialmente roedores), aves e lagartos, envolvendo o corpo da presa, fazendo constrição e por sufocamento, matando a presa. Sua boca é muito dilatável, e apresenta dentição áglifa - uma sequência de dentes simétricos, maciços e sem sulcos longitudinais ou ranhuras, tanto na arcada superior quanto inferior, o que é típico da Família Boidae (GOULART, 2004).

Entre os métodos de estabilização de fraturas mandibulares em cães e gatos se destacam a utilização de fio metálico, pino intramedular, fixador esquelético externo, resina acrílica e placa de compressão dinâmica (LEGENDRE, 2005; VERSTRAETE, 2007). A utilização de placas ósseas convencionais em fraturas de mandíbula está relacionada a diversas complicações, como a necessidade de grande elevação dos tecidos moles para sua aplicação, alto risco de lesão às raízes dentárias e aos vasos e nervos presentes no canal 
medular, além da difícil modelagem da placa na mandíbula, o que pode acarretar em má oclusão (MARRETTA, 1998). O desenvolvimento de miniplacas de diferentes formas e comprimentos possibilitou a adaptação dos princípios da fixação interna a ossos pequenos e delicados da face, o que revolucionou o tratamento das fraturas maxilofaciais em pessoas (BOUDRIEAU, 2004). Entretanto, há poucos relatos de sua aplicação em animais de companhia (GOMES et al., 2010).

O método apropriado para tratamento das fraturas mandibulares e maxilares é baseado no escore de avaliação e na localização da fratura (JOHNSON, 2008). Fraturas no corpo mandibular podem ser reduzidas com uma técnica específica ou técnicas combinadas, pois a linha de fratura pode estar sujeita a diferentes tipos de força. A margem gengival do osso é sobrecarregada em tensão, e a cortical ventral é sobrecarregada em compressão. Já fraturas no ramo mandibular apresentam grande cobertura muscular, proporcionando uma maior estabilidade dos fragmentos (PIERMATTEI; FLO; DECAMP, 2009).

Este artigo descreve a aplicação de placa de compressão dinâmica e parafusos na redução de fratura mandibular em uma serpente jiboia (Boa constrictor).

\section{Relato do caso}

Uma jiboia do sexo feminino, com peso de 8,0 $\mathrm{kg}$ e comprimento de $1,80 \mathrm{~m}$, foi encaminhada à Fundação Jardim Zoológico da Cidade do Rio de Janeiro - RIOZOO, após apreensão e resgate pelo Corpo de Bombeiros Militar do Estado do Rio de Janeiro. Ao chegar à fundação, a serpente não apresentava apreensão, observado após a captura e morte de um roedor fornecido como alimento. Durante o exame físico percebeu-se crepitação na região mandibular, e uma radiografia do crânio evidenciou a presença de fratura completa transversa no corpo mandibular direito. Decidiu-se então pela osteossíntese com uso de placa de compressão dinâmica e parafusos, sendo a intervenção cirúrgica realizada no dia seguinte ao exame físico, com a paciente permanecendo em jejum desde a captura.

Como medicação pré-anestésica foi utilizado cloridrato de cetamina $\left(20,0 \mathrm{mg} \cdot \mathrm{kg}^{-1}\right)$, midazolam $\left(1 \mathrm{mg} \cdot \mathrm{kg}^{-1}\right)$ e cloridrato de tramadol $\left(1,0 \mathrm{mg} \cdot \mathrm{kg}^{-1}\right)$, administrados pela via intramuscular (IM), aguardando-se 40 minutos para subsequente entubação com sonda endotraqueal 3.0, sem manguito. A manutenção anestésica foi realizada com isofluorano vaporizado em oxigênio a $100 \%$ (Figura 1A-B) ao efeito, com ventilação controlada e volume corrente de $50,0 \mathrm{~mL} \cdot \mathrm{kg}^{-1}$ e frequência de quatro respirações por minuto.

Com o diagnóstico radiológico (Figura 1C), a paciente em plano anestésico foi posicionada em decúbito lateral esquerdo, sendo feita incisão da pele na base do terço médio do corpo da mandíbula direita, seguida de divulsão da musculatura e exposição do foco da fratura, com auxílio de elevador periosteal (Figura 1D).

Dois orifícios foram feitos com perfuratriz e broca ortopédica de $1,5 \mathrm{~mm}$, transpassando-se a seguir um fio de náilon 2.0 para reduzir e estabilizar o foco da fratura (Figura 1E). Com a mesma broca foram perfurados dois orifícios para inserção dos parafusos de 2,0 mm x 0,6 mm usados para fixação da placa de 2,0 mm com três furos. Empregou-se um parafuso em cada segmento da fratura, deixando o furo central da placa no foco da fratura (Figura 1F).

A musculatura foi aproximada com pontos simples, usando fio poliglactina 9104.0 da mesma forma que o tecido subcutâneo, e a pele foi suturada com pontos simples separados, usando fio nylon 4.0 (Figura $1 \mathrm{G}$ ). A radiografia realizada imediatamente após a intervenção mostrou correto realinhamento ósseo e posicionamento adequado da placa e dos parafusos (Figura $1 \mathrm{H})$.

A recuperação anestésica ocorreu oito horas após a suspensão do fornecimento de isoflurano. No período pós-operatório, a serpente foi alojada 
em caixa plástica compatível com seu tamanho, gentamicina (sete administrações IM de 5,0 mg. $\mathrm{kg}^{-1}$ sendo medicada com meloxicam (0,2 mg. $\mathrm{kg}^{-1}$ a a intervalos de 72 horas) e enrofloxacina (10 cada 24 horas, durante 5 dias, por via subcutânea), administrações IM de $10 \mathrm{mg} \cdot \mathrm{kg}^{-1}$ a cada 48 horas).

Figura 1. Jiboia (Boa constrictor) do sexo feminino, com peso de $8,0 \mathrm{~kg}$ e comprimento de $1,80 \mathrm{~m}$. A. Paciente entubada e conectada ao circuito anestésico. B. Vista frontal da paciente entubada, com a cabeça em decúbito dorsal. C. Imagem radiográfica ventrodorsal, notando-se a presença de fratura transversa (seta). D. Imagem da linha de fratura no corpo mandibular (seta). E. Imagem do sítio cirúrgico após alinhamento e estabilização parcial com fio mononáilon 2.0 (seta). F. Placa de compressão dinâmica de 2,0 $\mathrm{mm}$ e dois parafusos de 2,0 $\mathrm{mm}$ x 0,6 mm colocados para estabilização da fratura. G. Ao final da intervenção cirúrgica observa-se a sutura de pele com fio mononáilon 4-0 (seta). H. Imagem radiográfica após aplicação da placa e dos parafusos, notando-se alinhamento ósseo adequado.

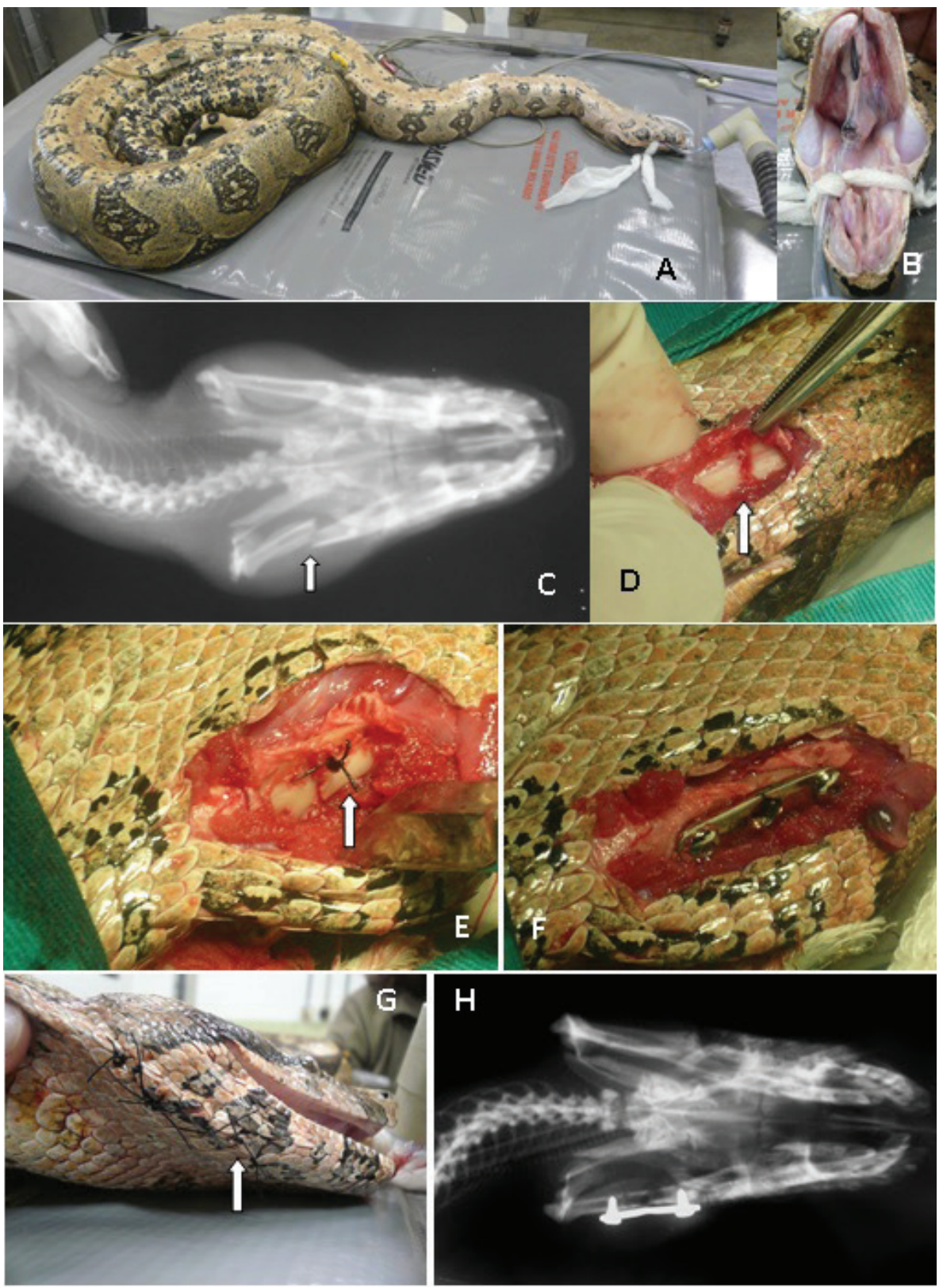

Fonte: Elaboração dos autores. 
Após a cirurgia, a paciente foi mantida em jejum por duas semanas, e a seguir passou a ser alimentada a cada sete dias com alimento industrializado pastoso para gatos diluído em solução de cloreto de sódio a $0,9 \%$, administrado por sondagem orogástrica em volume equivalente a 3,0\% do peso do animal, durante três meses. Depois disso, a dieta passou a ser constituída por um camundongo adulto vivo, servido a cada sete dias.

Aplicação tópica de iodo-polivinil-pirrolidona e spray de rifampicina sódica foi feita a cada 48 horas, durante 20 dias, e as suturas de pele foram removidas 45 dias após a intervenção.

\section{Discussão}

Segundo Athayde (2007), traumatismos provocados pelo homem em animais selvagens são frequentes, e essas agressões são devidas ao aparecimento desses animais em áreas urbanas, geralmente em função das alterações ambientais provocadas pelo desmatamento e uso econômico de suas áreas de habitat. Isso causa o desaparecimento das presas usuais e força esses animais, inclusive serpentes, a migrar para áreas urbanizadas, tornando-se vulneráveis a lesões provocadas por agressões, atropelamentos e acidentes de captura.

A indicação do tipo de tratamento para fraturas, seja ele conservador ou cirúrgico, tem sido baseada nos chamados critérios de estabilidade. A instabilidade implica na tendência a desvios adicionais e possível lesão neurológica, caso a fratura não seja imobilizada. Geralmente indica-se o uso de placas ortopédicas para estabilização de estruturas ósseas (PIERMATTEI; FLO; DECAMP, 2009).

A paciente deste relato apresentava fratura fechada no corpo da mandíbula, região que de acordo com Johnson (2008) é mais propensa a esse tipo de lesão, em função de sua grande extensão. Entretanto, o mesmo autor cita que a maioria das fraturas mandibulares dos animais é aberta, o que não foi observado neste caso.

A utilização de fio de náilon e placa com 3 furos foi suficiente para alinhar e manter estável o foco da fratura, corroborando a afirmação de Legendre (2005), de que em alguns casos de fraturas mandibulares a fixação rígida não é necessária, devido à não sustentação de peso. A paciente foi mantida em jejum por duas semanas, e a partir daí alimentada semanalmente por meio de sonda orogástrica durante três meses, o que evitou movimentação excessiva das hemiarcadas dentárias inferiores e consequente geração de forças atuantes no foco da fratura.

Serpentes macrostomatas apresentam a sínfise mandibular livre, ou seja, nestes animais, a região sinfisial pode ser composta por até quatro elementos, dois fibrosos e dois cartilaginosos. Por possuírem esta sínfise mandibular livre, a ingestão das presas é realizada através de uma série de movimentos unilaterais da mandíbula e da região palato-maxilar, característica essa que, impede que esses pacientes se alimentem de forma adequada com fraturas mandibulares (HOCKNULL, 2002). Mesmo não sendo realizados testes específicos de força com a paciente no que diz respeito à capacidade de apreensão de alimentos, observamos que a mesma matou um roedor e não conseguiu ingerilo; diante disso e do exame, ela foi encaminhada para intervenção cirúrgica no dia seguinte do atendimento. Contudo, os autores acreditam que a mesma teria dificuldades na apreensão do alimento se não fosse realizado a intervenção cirúrgica, entretanto, após 14 semanas da intervenção cirúrgica, quando a dieta passou a ser constituída por um camundongo adulto vivo, servido a cada sete dias, a serpente não apresentou qualquer tipo de dificuldade na apreensão e deglutição da presa.

Segundo Marretta (1998), uma das principais complicações pós-operatórias nas osteossíntese mandibulares em cães e gatos é a má-oclusão, fato que não foi observado nesta serpente, que conseguiu se alimentar normalmente quando passou a receber 
presa viva. Independente do tipo de alimentação, ela foi fornecida a cada sete dias, corroborando a afirmação de Cubas, Silva e Catão-Dias (2007) de que sete dias é o intervalo ideal para a alimentação desse tipo de serpentes.

Seis meses após a intervenção, nova avaliação radiográfica mostrou que havia formação de calo ósseo e que os parafusos pareciam frouxos, sendo então realizada sua remoção (Figura 2AB). Optou-se por não remover a placa, que se encontrava incorporada á mandíbula, sendo que sua retirada poderia predispor a fragilização ou nova fratura. Isso não interferiu no manejo diário e na sobrevida da paciente - examinada 18 meses após o procedimento cirúrgico, a jiboia apresentava bom estado nutricional e plena adaptação à vida em cativeiro no zoológico.

Figura 2. Imagens radiográficas do crânio da jiboia (Boa constrictor) mostrada na Figura 1, seis meses após a osteossíntese mandibular. A. Projeção ventrodorsal, notando-se presença da placa ortopédica e consolidação óssea. B. Projeção lateral, notando-se presença da placa ortopédica de 2,0 $\mathrm{mm}$ sem os parafusos, e consolidação óssea.

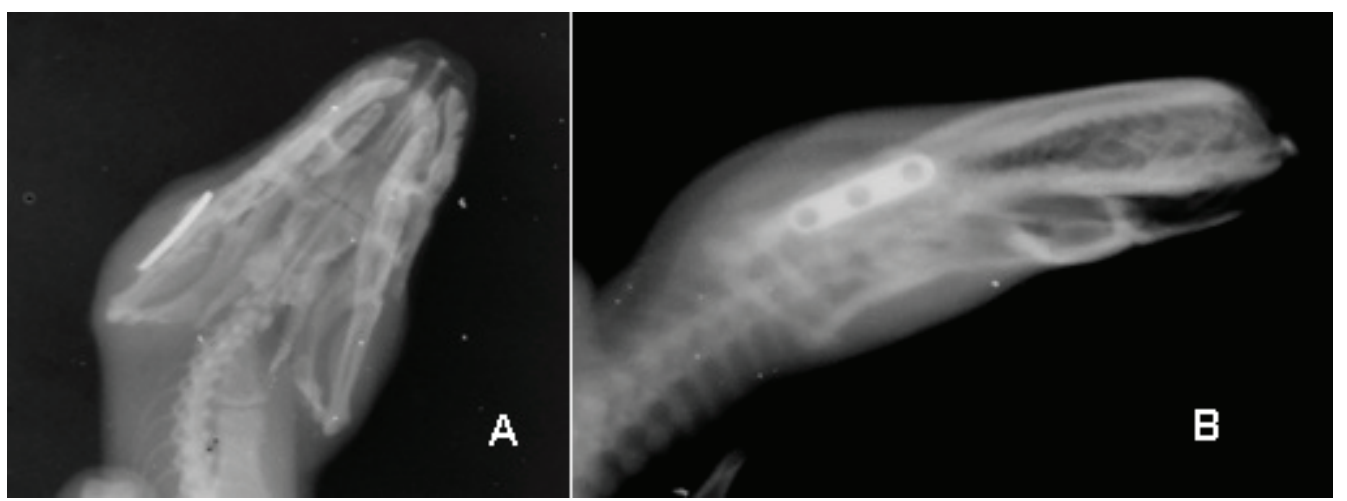

Fonte: Elaboração dos autores.

\section{Conclusões}

De acordo com o estado nutricional do animal e a linha de fratura observada ao exame radiográfico, concluiu-se que a lesão era recente, e provavelmente devida a falha no procedimento de contenção e manejo, no momento da captura para resgate.

A restrição alimentar inicial foi de grande valia, pois evitou sobrecarga no foco da fratura. O emprego de placa de compressão dinâmica e parafusos foi eficaz neste caso, proporcionando alinhamento e estabilidade suficientes para um resultado satisfatório, possibilitando que a serpente atualmente seja capaz de capturar uma presa, apreendê-la na boca e ingeri-la sem mostrar qualquer complicação pós-operatória.

\section{Referências}

ATHAYDE, G. C. Tratamento de lesões traumáticas em sucuri (Eunectes murinus). 2007. Trabalho de conclusão (Curso de Especialização em Clínica Médica e Cirúrgica em Animais Selvagens e Exóticos) - Universidade Castelo Branco, Rio de Janeiro.

BOUDRIEAU, R. J. Miniplate reconstruction of severely comminuted maxillary fractures in two dogs. Veterinary Surgery, Davis, v. 33, n. 2, p. 154-163, 2004.

CUBAS, Z. S.; SILVA, J. C. R.; CATÃO-DIAS, J. L. Tratado de animais selvagens. São Paulo: Roca, 2007. $1354 \mathrm{p}$.

GOMES, C.; GOUVÊA, A. S.; ALIEVI, M. M.; CONTESINI, E. A.; PIPPI, N. L. Miniplacas de titânio na redução de fraturas mandibulares em cães e gatos: estudo de seis casos. Ciência Rural, Santa Maria, v. 40, n. 5, p. 1128-1133, 2010.

GOULART, C. E. S. Herpetologia, herpetocultura e medicina de répteis. Rio de Janeiro: L.F. livros, 2004. 330 p. 
HOCKNULL, S. A. Comparative maxillary and dentrary morphology of the australian dragons (Agamidae: Squamata): a framework for fossil identification. Memoirs of the Queensland Museum, Brisbane, v. 48, n. 1, p. 125-145, 2002.

JOHNSON, A. L. Tratamento de fraturas específicas. In: FOSSUM, T. W. Cirurgia de pequenos animais. 4. ed. São Paulo: Elsevier, 2008. p. 1015-1142.

LEGENDRE, L. Maxillofacial fracture repairs. Veterinary Clinics of North America. Small Animal Practice, Philadelphia, v. 35, n. 4, p. 985-1008, 2005.

MARRETTA, S. M. Maxillofacial surgery. Veterinary Clinics of North America. Small Animal Practice, Philadelphia, v. 28, n. 5, p. 1285-1295, 1998.
PIERMATTEI, D. L.; FLO, G.; DECAMP, C. E. Ortopedia e tratamento de fraturas de pequenos animais. 4. ed. São Paulo: Manole, 2009. 896 p.

RAIMUNDO, R. C.; GUERRA, L. A. P.; ANTUNES, A. A.; CARVALHO, R. W. F.; SANTOS, T. S. Fraturas de mandíbula: análise retrospectiva de 27 casos. Revista de Cirurgia e Traumatologia Buco-maxilo-facial, Recife, v. 8, n. 1, p. 57-62, 2008.

VERSTRAETE, F. J. M. Fraturas maxilofaciais. In: SLATTER, D. Manual de cirurgia de pequenos animais. 3. ed. São Paulo: Manole, 2007. p. 2190-2207. 
